\title{
ON A CLASS OF PUNCTUAL CONVEX FUNCTIONS
}

\author{
Aurelia Florea AND EUGEN PĂLTĂNEA
}

Abstract. The aim of this paper is to show that the inequality of Jensen for real functions holds under a weaker condition than the usual convexity on an interval. Thus, we introduce the concept of convexity at a point. We present and discuss the basic properties of the class of functions satisfying the punctual convexity. This concept is further extended to the lateral convexity at a point. The interest in these notions is the extensions of some inequalities, as illustrated in this paper. It should be noted that the usual convexity on intervals does not provide a direct answer for these problems.

Mathematics subject classification (2010): 26A51, 26B25, 26D10. a point.

Keywords and phrases: Convex functions, concave functions, convexity at a point, lateral convexity at

\section{REFERENCES}

[1] P. CZINDER AND Z. PÁles, An extension of the Hermite-Hadamard inequality and an application for Gini and Stolarsky means, J. Inequal. Pure and Appl. Math. 5 (2004), art. 42.

[2] A. Florea AND C. P. Niculescu, A Hermite-Hadamard inequality for convex-concave symmetric functions, Bull. Soc. Sci. Math. Roum. 50 (2007), 149-156.

[3] F. C. Minuţă, Point convexity, Ann. Univ. of Craiova, Math. and Comp. Sc. Series 37 (2010), 100 105.

[4] C. P. Niculescu And L.-E. Persson, Convex Functions and their Applications: A Contemporary Approach, CMS Books in Mathematics, Springer-Verlag New York Inc., New York, 2006.

[5] C. P. Niculescu AND C. I. SPIRIDON, New Jensen-type inequalities, arXiv:1207.6877 (2012), http://arxiv.org/abs/1207.6877. 